\title{
ASOCIACIÓN DE LAS CARRERAS DE CIENCIAS, CIENCIAS ECONOMICAS Y CIENCIAS SOCIALES CON EL PERFIL DE INGRESO DE SUS ESTUDIANTES
}

\author{
RELATION OF CAREERS OF SCIENCE, ECONOMICS AND SOCIAL \\ SCIENCES WITH THE ENTRANCE PROFILE OF STUDENTS
}

\section{Luis Rojas Torres*}

\begin{abstract}
RESUMEN
Este estudio se realizó con el objetivo de asociar las carreras de las Facultades de Ciencias, Ciencias Económicas y Ciencias Sociales de la Universidad de Costa Rica, con un perfil de ingreso de sus estudiantes, basado en el promedio de admisión y la opción que seleccionaron para la carrera a la que ingresaron, durante el año 2013. Mediante un análisis de correspondencias se observó que las carreras en la Facultad de Ciencias Sociales y Ciencias Económicas se ubican en tres grupos distanciados entre sí, asociados a diferentes niveles del promedio de admisión. Las carreras de la Facultad de Ciencias se asocian entre ellas y se alejan de los perfiles, indicando poca asociación con estos; en esta facultad se presenta el caso de que la carrera de Química se asocia con un perfil de ingreso en segunda opción. Por otro lado, se muestra que en las carreras asociadas a perfiles altos, los porcentaje de no matrícula son inferiores al 12\%, mientras que en los de perfiles bajos es superior al $20 \%$. Estos resultados brindan un marco referencial sobre un problema del sistema de admisión universitaria asociado a las carreras con perfiles de bajo ingreso.
\end{abstract}

PALABRAS CLAVE: ESTUDIANTE UNIVERSITARIO * ADMISIÓN EN LA UNIVERSIDAD * ELECCIÓN DE CARRERA * CIENCIAS SOCIALES * CIENCIAS ECONÓMICAS * CIENCIAS

\section{ABSTRACT}

This study was carried in order to associate the careers of the Faculties of Sciences, Economics Sciences and Social Sciences of University of Costa Rica with a profile of income of its students, based on the average of admission and the option they selected the career to which entered. By an analysis of correspondence, it was noted that the careers at the Faculty of Economics Sciences and Social Sciences are located in three groups distanced each other, associated with different average levels of admission. Careers of the Faculty of Science are associated with each other and deviate from the profiles, indicating little association with these; this faculty presents the case that Chemistry career is associated with a profile of income in second option. Also, it shows that in careers associated with

\footnotetext{
* Instituto de Investigaciones Psicológicas (IIP) de la Universidad de Costa Rica (UCR).
} luismiguel.rojas@ucr.ac.cr 
high profiles, the percentage of non-registration is less than $12 \%$, while in the low profiles it is exceeding $20 \%$. These results provide a frame of reference on a problem of the college admissions system associated with races with low income profiles.

KEYWORDS: UNIVERSITY STUDENT * UNIVERSITY'S ADMISSION * OCCUPATIONAL CHOISE * SOCIAL SCIENCES * ECONOMICS SCIENCES * SCIENCES

\section{INTRODUCCIÓN}

El proceso de admisión a la Universidad de Costa Rica (UCR) a manera general, consiste en un algoritmo en el cual se le va asignando carrera a los solicitantes de ingreso a la ucr, según su promedio de admisión ${ }^{1}$; con la salvedad de que si un aspirante solicitó ingresar a una carrera que ya había sido llenada con aspirantes con promedios de admisión más altos, puede ingresar a otra carrera que previamente había indicado como carrera en segunda opción (Reglamento del Proceso de Admisión mediante Prueba de Aptitud Académica, 2003).

Este modelo de admisión a la Universidad de Costa Rica, le ha traído a esta institución una serie de circunstancias problemáticas, entre las cuales se pueden mencionar: estudiantes admitidos en carreras para la cual no presentan interés o habilidad, estudiantes admitidos que nunca matriculan un curso, quitándole el campo a otro que si hubiera realizado matrícula; sobre representación de grupos de población e ingreso de personas a la Universidad con bajos indicadores de aptitud académica, mientras que personas con indicadores altos se quedan sin campo.

La primer situación que se plantea referente a estudiantes empadronados en carreras para las cuales no poseen interés o

$1 \quad$ El promedio de admisión es la media de a) la nota obtenida en el examen de admisión a la Universidad de Costa Rica (denominado Prueba de Aptitud Académica) y b) el promedio de las notas obtenidas en las materias básicas del último ciclo de educación secundaria. Los aspirantes a estudiar en una carrera son seleccionados por medio del promedio de admisión. El promedio de admisión se estandariza a una escala de 200 a 800 y se establece como requisito básico para ingresar a la UCR, el cual se encuentra en el rango de 442 a 800 . habilidad, se ve reflejado en Rivera (2013), cuando menciona que tres de cada diez nuevos estudiantes de la Universidad de Costa Rica desean cambiarse de carrera.

Por otro lado, en un cuestionario aplicado a los estudiantes de los cursos introductorios de Filología, Estadística, Física, Meteorología, Química y Ciencias del Movimiento Humano en el 2012, se obtuvo que el 39\% de los estudiantes de la muestra se encontraban empadronados en carreras para la cual no tienen interés. Además, en este conjunto de estudiantes, el 86\% manifiesta que se encuentra en esas carreras debido a situaciones asociadas al modelo de admisión. La situación se agrava cuando se analizan las carreras por separado, ya que en Química y Filología, más del $60 \%$ de sus estudiantes no manifiestan interés por estas (Programa de Pruebas Específicas, 2012).

Con respecto a la habilidad para cursar una carrera, se menciona que en los estudiantes de nuevo ingreso en el 2012, de las carreras de Estadística, Física, Meteorología y Química, solo el 30\% de los admitidos poseían un nivel de habilidad cuantitativa medio o alto, lo cual es preocupante, ya que esta habilidad es necesaria para desempeñarse en estas carreras (Proyecto de Habilidades Cuantitativas, 2012).

El segundo problema mencionado, es el gran porcentaje de estudiantes que ingresan a la Universidad que deciden no realizar matrícula. Para el 2013, el porcentaje de "no matrícula" fue de un $21,53 \%$, esto indica que uno de cada cinco estudiantes admitidos en la ucr, está desaprovechando un campo que probablemente otro estudiante desearía utilizar (Oficina de Registro e Información, 2013).

En cuanto a la sobre representación de estudiantes, en Montero (2013) se evidencia que las personas del quintil más alto de ingresos $y$ 
provenientes de colegios privados, ingresan a la Universidad en una proporción más alta de la que representan a nivel nacional. En el caso de los estudiantes provenientes de colegios privados, se tiene que estos representan el 19,1\% de aspirantes a ingresar a la ucR; sin embargo, el $35,2 \%$ de nuevos ingresos son provenientes de este grupo, lo cual indica que la proporción de estudiantes de colegios privados en la Universidad es mayor al doble de la proporción de estos a nivel nacional. Lo anterior provoca que haya una subrepresentación de otros grupos como los estudiantes de colegios públicos o de los quintiles de más bajo ingreso.

El último problema presentado está asociado al ingreso de personas con promedios de admisión bajos (lo cual representa probabilidades bajas de éxito académico) (Jiménez y Morales, 2009- 2010; Rojas, 2013), mientras que personas con probabilidades altas de éxito académico se quedan sin campo. Este problema tiene su origen en que los aspirantes con promedios de admisión bajos solicitan carreras de poca demanda, con el fin de asegurarse un campo en la Universidad (denominados grupo intensión), mientras que aspirantes con promedios altos solicitan carreras a las cuales si desean ingresar $y$ que son de alta demanda (denominados grupo pretensión), por lo cual pueden quedarse sin cupo (Vicerrectoría de Vida Estudiantil, 2013).

Los problemas anteriores son solo un matiz de las circunstancias que se ven reflejadas inmediatamente después del proceso de admisión, lo cual se agrava cuando se contemplan las consecuencias que traerán a la Universidad al pasar de los años.

Debido a las razones anteriores, se considera necesario elaborar un perfil de los estudiantes de cada carrera, basado en las condiciones del proceso de admisión que los llevaron a ingresar a la carrera en la cual fueron admitidos. Además, esto permite estudiar relaciones de los problemas planteados con las características del perfil de ingreso de los estudiantes de cada carrera.

En particular, resulta importante trabajar con las Facultades de Ciencias, Ciencias
Económicas y Ciencias Sociales, porque son de las unidades académicas que presentan mayores problemas en consolidación de matrícula $y$ en recibimiento de población de estudiantes del grupo intensión (Vicerrectoría de Vida Estudiantil, 2013).

\section{MATERIALES Y MÉTODO}

\subsection{OBJETIVOS Y METODOLOGÍA}

El objetivo general de este trabajo es asociar las carreras de las Facultades de Ciencias, Ciencias Económicas y Ciencias Sociales con un perfil de ingreso de sus estudiantes. Para su consecución se proponen los siguientes objetivos específicos: a) diseñar una variable categórica asociada al perfil de ingreso de los estudiantes, b) determinar conjuntos de carreras asociadas a cada categoría del perfil de ingreso y c) estudiar la asociación de estas agrupaciones con una situación problemática de la admisión universitaria.

El primer objetivo se trabaja basándose en el promedio de admisión obtenido por los estudiantes y la opción en que seleccionaron la carrera en la cual fueron admitidos; el segundo objetivo se abarca describiendo las asociaciones de las carreras según facultad, con las categorías del perfil de ingreso, mediante el uso de la técnica estadística Análisis de Correspondencias y finalmente, el último objetivo se logra mediante el estudio de la asociación de los porcentajes de estudiantes que realizan matrícula con el perfil de ingreso de los estudiantes a las carreras.

\subsection{DATOS}

Para este trabajo se utilizaron los datos de matrícula de la Universidad de Costa Rica en el recinto Rodrigo Facio para el año 2013, los cuales fueron proporcionados por la Oficina de Registro e Información. La muestra utilizada estuvo compuesta por todos los estudiantes de primer ingreso, admitidos a las carreras de las Facultades de Ciencias Económicas, Ciencias Sociales y Ciencias. 
CUADRO 1

DISTRIBUCIÓN DEL PERFIL DE INGRESO DE LOS ESTUDIANTES ADMITIDOS

EN LAS FACULTADES DE CIENCIAS, CIENCIAS ECONÓMICAS Y CIENCIAS SOCIALES

SEGÚN CARRERA, 2013

\begin{tabular}{|c|c|c|c|c|c|c|}
\hline \multirow[b]{2}{*}{ CARRERA } & \multicolumn{5}{|c|}{ PERFIL DE INGRESO DE LOS ESTUDIANTES } & \multirow[b]{2}{*}{ TOTAL } \\
\hline & $\begin{array}{c}\text { BAJOS } \\
\text { OPCIÓN1 }\end{array}$ & $\begin{array}{c}\text { BAJOS } \\
\text { OPCIÓN2 }\end{array}$ & $\begin{array}{l}\text { MEDIOS } \\
\text { OPCIÓN1 }\end{array}$ & $\begin{array}{l}\text { MEDIOS } \\
\text { OPCIÓN2 }\end{array}$ & ALTOS & \\
\hline Biología & 0 & 0 & 32 & 11 & 11 & 54 \\
\hline Física & 11 & 12 & 32 & 32 & 12 & 99 \\
\hline Meteorología & 29 & 60 & 3 & 7 & 0 & 99 \\
\hline Geología & 19 & 14 & 27 & 18 & 2 & 80 \\
\hline Matemática & 5 & 10 & 10 & 12 & 7 & 44 \\
\hline Actuariales & 4 & 2 & 23 & 3 & 8 & 40 \\
\hline Química & 0 & 0 & 25 & 31 & 9 & 65 \\
\hline Estadística & 1 & 1 & 41 & 29 & 3 & 75 \\
\hline Dir. Empresas & 0 & 0 & 206 & 140 & 14 & 360 \\
\hline Contaduría & 40 & 38 & 63 & 48 & 1 & 190 \\
\hline Adm. Pública & 21 & 23 & 30 & 45 & 1 & 120 \\
\hline Adm. Aduanera & 0 & 0 & 59 & 15 & 14 & 88 \\
\hline Economía & 0 & 0 & 37 & 5 & 34 & 76 \\
\hline Comunicación & 0 & 0 & 21 & 1 & 56 & 78 \\
\hline Psicología & 0 & 0 & 68 & 18 & 14 & 100 \\
\hline Políticas & 16 & 12 & 41 & 52 & 4 & 125 \\
\hline Trabajo social & 0 & 0 & 47 & 32 & 1 & 80 \\
\hline Historia & 26 & 37 & 16 & 6 & 0 & 85 \\
\hline Archivística & 10 & 18 & 7 & 13 & 0 & 48 \\
\hline Geografía & 26 & 36 & 9 & 4 & 0 & 75 \\
\hline Antropología & 24 & 20 & 27 & 13 & 6 & 90 \\
\hline Sociología & 21 & 25 & 29 & 15 & 0 & 90 \\
\hline TOTAL & 253 & 308 & 853 & 550 & 197 & 2161 \\
\hline
\end{tabular}

Fuente: Oficina de Registro e Información de la Universidad de Costa Rica.

Las carreras de la Facultad de Ciencias Económicas son: Estadística, Dirección de Empresas, Contaduría Pública, Administración Pública, Administración Aduanera y Economía; las de la Facultad de Ciencias Sociales son: Comunicación Colectiva, Psicología, Ciencias Políticas, Trabajo Social,
Historia, Archivística, Geografía, Antropología y Sociología; mientras que en la Facultad de Ciencias las carreras son: Biología, Física, Meteorología, Matemática, Ciencias Actuariales y Química.

Para conformar la variable asociada al perfil de ingreso de los estudiantes, se definieron 
cinco grupos basados en el promedio de admisión a la Universidad de Costa Rica y en el orden de prioridad en que el estudiante seleccionó la carrera a la que fue admitido.

Las personas con promedios de admisión entre 442 y 560, se les denominó bajos y se clasificaron en dos grupos, los que entraron a la carrera que tenían como primera opción (bajos_opción1) y los que ingresaron a su segunda alternativa (bajos_opción2). A los estudiantes con promedios mayores o igual a 560 y menores a 680 se les asignó el nombre de medios y se clasificaron, al igual que los bajos, en medios_opción1 y medios_opción2. Los estudiantes con promedios mayores a 680 se les denominaron altos y no se dividieron según opción de carrera, ya que la mayoría ingresan a la carrera que seleccionan como prioridad.

En el cuadro 1, se presenta la tabla de contingencia entre las carreras de las Facultades estudiadas y las categorías del perfil de los estudiantes. Se puede apreciar que en las carreras de Geografía, Historia y Meteorología, la categoría con más estudiantes es la de bajos_opción2, contrario a Comunicación, en la cual la categoría altos es la que presenta mayor número.

\subsection{MÉTODO}

La técnica estadística que se utiliza en este trabajo es el Análisis de Correspondencias simples, la cual es "una técnica multivariante que se centra en describir afinidades entre las categorías de dos variables cualitativas para luego representarla en un espacio de pequeña dimensión que permita interpretar, por un lado, las similitudes entre las categorías de una variable $y$, por otro lado, las relaciones entre las categorías de ambas variables" (Cabrera, 2008: 22).

Se puede decir que este es un método de análisis de la relación entre variables categóricas, que posibilita visualizar en un gráfico las asociaciones entre las categorías de estas variables, ya que estas resultan difíciles de observar en tablas de contingencia muy grandes. Los ejes de este gráfico son las dos dimensiones que explican el mayor porcentaje de la variancia (en el caso de trabajar con dos dimensiones, se puede realizar con un mayor número), que se presenta en la relación entre las dos variables estudiadas.

Para este análisis se utilizó el proceso de normalización denominado simétrica y la medida de distancia Chi Cuadrado, el software utilizado fue el Statistical Package for Social Sciences 20.0 (spss).

\section{RESULTADOS}

\subsection{CIENCIAS}

En el análisis de correspondencias se observó que con dos dimensiones se logra explicar el 94\% de la variancia de las carreras de la Facultad de Ciencias con las categorías de la variable perfil de los estudiantes. En el gráfico 1 se presenta la ubicación de las carreras y las categorías en un plano coordenado sobre las dimensiones establecidas. Para una mejor interpretación de los resultados de este gráfico $y$ los posteriores, se construyeron círculos que agruparon los puntos más cercanos. Los detalles de la inercia, la masa y la contribución de los puntos en las dimensiones se presentan en los anexos.

Se puede notar en el gráfico, que los puntos se distribuyeron muy separados entre sí, a diferencia de lo que se verá en las otras dos facultades. Se distinguieron cinco agrupaciones: a) Química y medios_opción2, b) Física, Matemática y Geología, c) Meteorología, bajos_opción1 y bajos_opción2, d) Biología, altos, medios_opción1 y e) Actuariales de manera solitaria.

Se puede decir que Meteorología se asocia a un perfil bajo de ingreso de sus estudiantes, mientras que el de Química a uno medio en segunda opción y el de Biología a perfiles altos $y$ medios en primera opción. Las carreras de Matemática, Física, Geología y Actuariales no estuvieron cerca de ningún perfil de ingreso, por lo cual no se puede concluir mucho sobre el perfil de sus estudiantes. 
GRÁFICO 1

UBICACIÓN DE LAS CARRERAS DE CIENCIAS Y LAS CATEGORÍAS DE PERFIL DE LOS ESTUDIANTES EN LAS DIMENSIONES DEL ANÁLISIS DE CORRESPONDENCIA

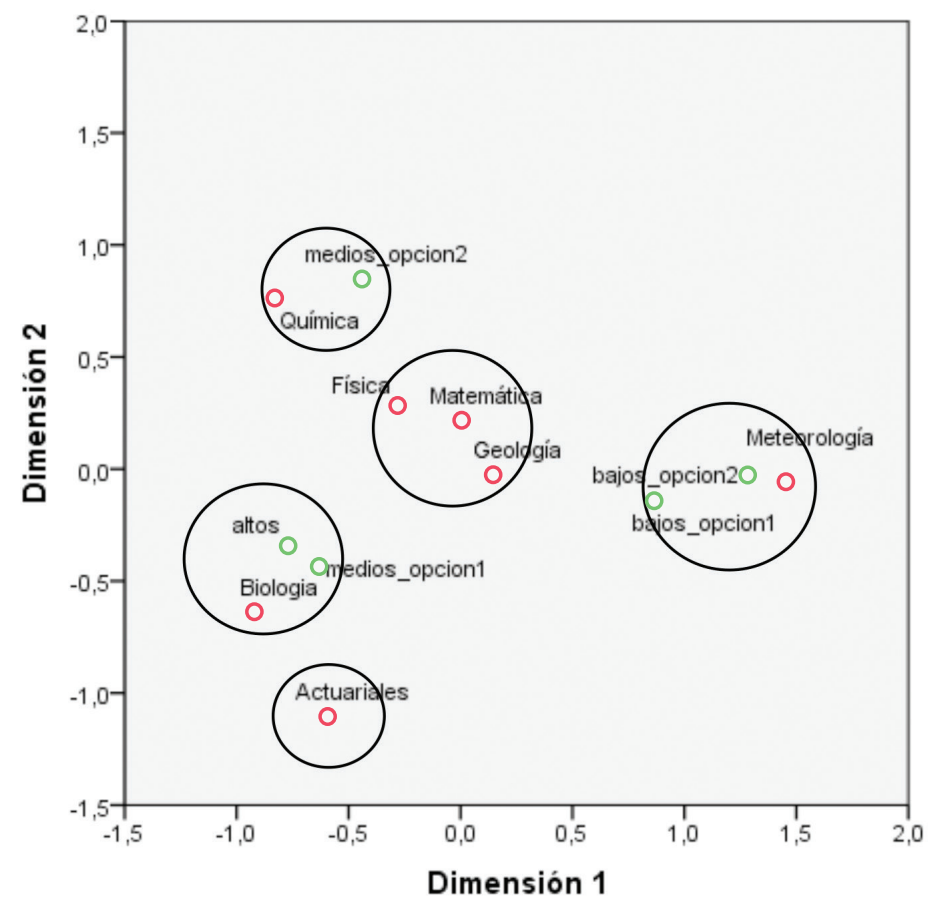

O carrera_estudio

Opromedio_opcion1

Nota: Porcentaje de variancia explicada por la primera dimensión $=.611$, por la segunda dimensión $=.328$ y por las dos dimensiones en conjunto $=.940$

\subsection{CIENCIAS ECONÓMICAS}

Para la relación entre las carreras de Ciencias Económicas y las categorías del perfil de los estudiantes, se obtuvo que el análisis de correspondencias con dos dimensiones explicó un $94,5 \%$ de la variabilidad de esta asociación. En el gráfico 2 se presenta la ubicación de estas carreras y categorías, en el plano coordenado por estas dos dimensiones.

Se distinguieron tres agrupaciones principales, la primera conformada por bajos_opción1, bajos_opción2, Administración Pública y Contaduría, por lo cual se puede concluir que para estas carreras el perfil de ingreso es bajo.

Otra agrupación estuvo conformada por Administración Aduanera, Estadística y Dirección de Empresas, medios_opción2 y medios_ opción1, por lo cual se puede decir que estas carreras tienen perfiles medios de ingreso.

Por otro lado, Economía y la categoría altos, conformaron un grupo muy distante de los otros dos y además, presentaron coordenadas muy similares entre sí, esto implica que el perfil de ingreso de Economía es alto y bastante diferenciado del resto de carreras de esta facultad. 

ESTUDIANTES EN LAS DIMENSIONES DEL ANÁLISIS DE CORRESPONDENCIAS

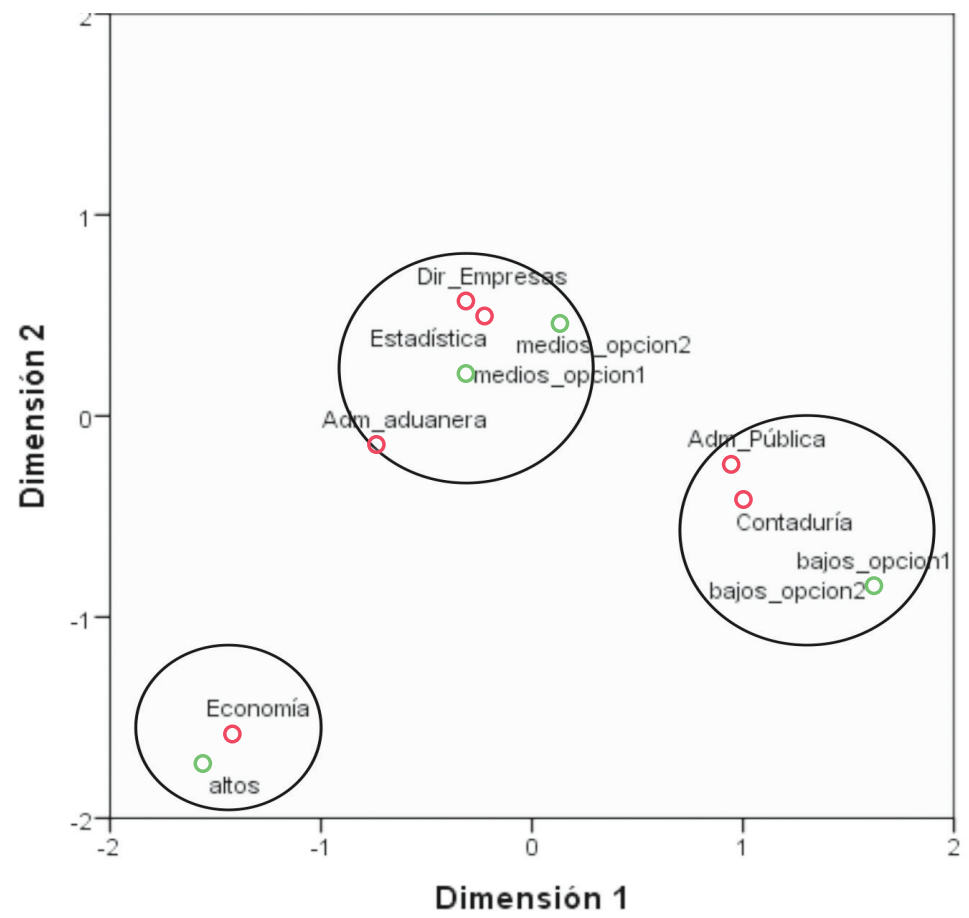

O carrera_estudio

promedio_opcion1

Nota: Porcentaje de variancia explicada por la primera dimensión $=.833$, por la segunda dimensión $=.113$ y por las dos dimensiones en conjunto $=.945$

\subsection{CIENCIAS SOCIALES}

Con respecto a la relación entre las carreras de la Facultad de Ciencias Sociales con el perfil de los estudiantes de nuevo ingreso, se obtuvo que el utilizar dos dimensiones, explica un $97,8 \%$ de la variancia de esta relación. En el gráfico 3 se presenta un plano coordenado para estas dos dimensiones, donde se ubican cada una de las carreras y las categorías del perfil de los estudiantes.

Al igual que en la Facultad de Ciencias Económicas, se distinguieron tres agrupaciones, donde las carreras se asociaron a categorías bajas, medias o altas. El grupo con carreras asociadas a perfiles bajos de ingreso, contuvo a bajos_opción1, bajos_opción2, Historia, Geografía, Archivística, Antropología y Sociología.

El grupo de perfiles de ingreso medio se conformó por medios_opción1, medios_ opción2 y las carreras de Trabajo Social, Psicología y Ciencias Políticas. Finalmente, de manera similar a lo observado en Ciencias Económicas, las coordenadas de la categoría altos y la carrera Comunicación Colectiva, fueron muy similares $y$ determinaron un grupo a una distancia considerable de los otros dos. 
GRÁFICO 3

UBICACIÓN DE LAS CARRERAS DE CIENCIAS SOCIALES Y LAS CATEGORÍAS DE PERFIL DE LOS ESTUDIANTES EN LAS DIMENSIONES DEL ANÁLISIS DE CORRESPONDENCIAS

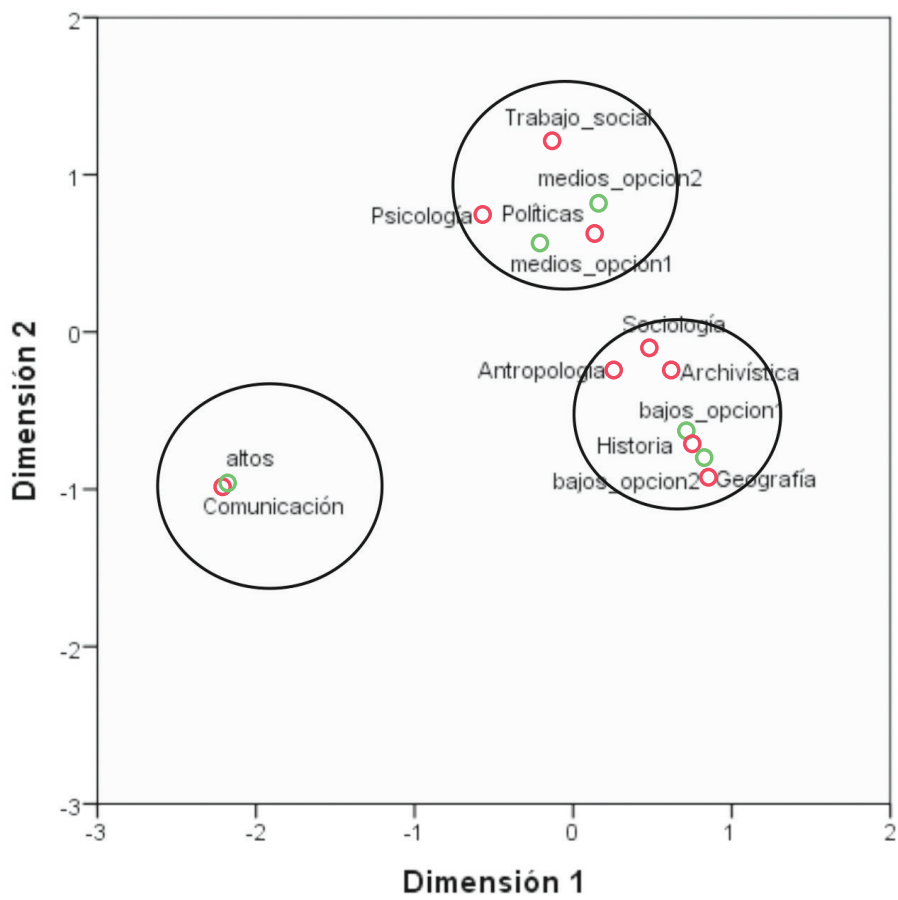

O carrera_estudio Opromedio_opcion1

Nota: Porcentaje de variancia explicada por la primera dimensión $=.664$, por la segunda dimensión $=.314$ y por las dos dimensiones en conjunto $=.978$

\subsection{ANÁLISIS DE LA MATRÍCULA EN ESTAS CARRERAS}

Como resultado complementario a los obtenidos en el análisis de correspondencias por Facultad, en el cuadro 2 se presenta la cantidad de estudiantes que realizaron matrícula en el primer semestre de 2013. El realizar matrícula implica que matricularon al menos un curso en la ucr, el cual puede que no esté en su plan de estudios.

De las ocho carreras que se asociaron a perfiles bajos de las tres facultades (Meteo- rología, Administración Pública, Contaduría, Historia, Geografía, Antropología, Archivística y Sociología), solo dos carreras (Sociología e Historia) tuvieron porcentajes de estudiantes que no realizaron matrícula (no matrícula) inferiores al $20 \%$.

En las carreras de grupos asociadas a perfiles medios (Trabajo Social, Psicología, Ciencias Políticas, Estadística, Administración Aduanera y Dirección de Empresas), solo Administración Aduanera, Psicología y Trabajo Social tuvieron porcentajes de no matrícula menores al $20 \%$. 
CUADRO 2

FRECUENCIAS DE REALIZACIÓN DE MATRÍCULA

SEGÚN CARRERA, 2013

\begin{tabular}{|c|c|c|c|c|}
\hline \multirow{2}{*}{ CARRERA } & \multicolumn{2}{|c|}{ NO REALIZÓ MATRÍCULA } & \multicolumn{2}{|c|}{ REALIZÓ MATRÍCULA } \\
\hline & ABSOLUTA & $\%$ & ABSOLUTA & $\%$ \\
\hline Meteorología & 25 & 25 & 74 & 75 \\
\hline Física & 28 & 28 & 71 & 72 \\
\hline Matemática & 15 & 34 & 29 & 66 \\
\hline Geología & 17 & 21 & 63 & 79 \\
\hline Actuariales & 4 & 10 & 36 & 90 \\
\hline Química & 7 & 11 & 58 & 89 \\
\hline Biología & 11 & 20 & 43 & 80 \\
\hline Contaduría & 49 & 26 & 141 & 74 \\
\hline Adm. Pública & 43 & 36 & 77 & 64 \\
\hline Estadística & 22 & 29 & 53 & 71 \\
\hline Dir. Empresas & 71 & 20 & 289 & 80 \\
\hline Adm. Aduanera & 14 & 16 & 74 & 84 \\
\hline Economía & 9 & 12 & 67 & 88 \\
\hline Comunicación & 7 & 9 & 71 & 91 \\
\hline Psicología & 16 & 16 & 84 & 84 \\
\hline Políticas & 32 & 26 & 93 & 74 \\
\hline Trabajo Social & 15 & 19 & 65 & 81 \\
\hline Historia & 15 & 18 & 70 & 82 \\
\hline Archivística & 10 & 21 & 38 & 79 \\
\hline Geografía & 23 & 31 & 52 & 69 \\
\hline Antropología & 20 & 22 & 70 & 78 \\
\hline Sociología & 14 & 16 & 76 & 84 \\
\hline TOTAL & 467 & 22 & 1694 & 78 \\
\hline
\end{tabular}

Fuente: Oficina de Registro e Información de la Universidad de Costa Rica.

En las carreras de Economía y Comunicación, que están asociadas a perfiles altos, se puede ver que los porcentajes de no matrícula no superan el 12\%. El caso de Biología, que está asociada a medios_opción1 y a altos, presenta un porcentaje de no matrícula del $20 \%$.

Las tres carreras de Ciencias que se agruparon entre sí, pero que no se agruparon con ninguna categoría del perfil de ingreso (Matemática, Física y Geología), presentan porcentajes de no matrícula superiores al $20 \%$, mientras que Actuariales que no se agrupó con nadie presenta un $10 \%$ de estudiantes que no realizaron matrícula.

\section{CONCLUSIONES}

Primeramente, el análisis de correspondencias permitió crear grupos de carreras similares entre sí, en cuanto a su asociación con los perfiles de los estudiantes. En las tres facultades 
con únicamente dos dimensiones, se logró explicar más del $94 \%$ de la variancia, lo cual indica que las ubicaciones presentadas en los gráficos, tanto de las carreras como de las categorías del perfil de los estudiantes, son bastante informativas.

Por otro lado, de todas las carreras analizadas únicamente, Química es una carrera asociada directamente a un perfil de ingreso en segunda opción, esto indica que los estudiantes de esta carrera probablemente no deseen estar en ella. Cuando se analizan los datos en bruto, se observa que casi el 50\% (31 de 65) de los estudiantes de esta carrera, entraron con promedios de admisión en segunda opción.

Luego, las carreras de Matemática, Física y Geología se agrupan entre ellas, pero no se ubican cerca de alguna de las categorías del perfil de ingreso, esto indica que tienen una asociación pequeña con estas, pero una alta entre ellas. Una de las similitudes entre estas carreras, son sus altos índices de no matrícula.

Ciencias Actuariales no se asoció con ningún punto del análisis y curiosamente es la carrera de la Facultad de Ciencias que presenta menor porcentaje de no matrícula (y la segunda de todas las carreras analizadas), habrá que realizar más estudios para determinar si esta razón justifica que no se haya agrupado con las otras tres carreras no asociadas a ningún perfil de ingreso.

Aparte de Matemática, Física y Geología, también Meteorología y Biología presentan índices de no matrícula bastante altos, por tanto se hace una llamada de atención sobre las situaciones que enfrenta la Facultad de Ciencias, al presentar cinco de siete carreras con problemas de no matrícula. Esto parece indicar que los estudiantes de esta facultad no quieren estudiar en ella, por tanto se debe diseñar propuestas de retención de estudiantes, además de elaborar mecanismos de selección que les permitan determinar que aspirantes realmente estudiarán en sus carreras.

$\mathrm{El}$ análisis de correspondencias permitió asignar perfiles de ingreso a todas las carreras de las facultades de Ciencias Sociales y Ciencias Económicas, los cuales se basaron prácticamente en el promedio de admisión. No se logró detectar en estas facultades carreras asociadas con una prioridad de selección, como si se evidenció en Ciencias con la carrera de Química.
En cuanto a los estudiantes que no realizan matrícula, se puede apreciar que en las carreras asociadas a perfiles bajos de ingreso en Ciencias Sociales, Ciencias Económicas y Ciencias, los porcentajes de estudiantes en esta condición son superiores al 20\%, lo cual puede ser un indicador de que estos entraron a esas carreras con el único propósito de asegurarse un campo en la Universidad, valiéndose de que para ingresar a ellas se requieren promedios de admisión relativamente bajos. Solamente Historia y Sociología son carreras asociadas a perfiles bajos de ingreso con porcentajes de no matrícula menores al $20 \%$, lo cual puede indicar que los estudiantes de estas carreras si deseen estudiarlas, queda pendiente investigar sobre este carácter distinto.

En las carreras asociadas a perfiles medios de ingreso la no matrícula se da en menor grado que en los grupos asociados a perfiles bajos, ya que solo tres de seis carreras fueron las que presentaron porcentajes de no matrícula mayores o iguales al $20 \%$, a diferencia de las carreras asociadas a perfiles bajos, donde esa condición se dio en dos de ocho carreras.

Finalmente, en las carreras asociadas a perfiles de ingreso altos, el porcentaje de no matrícula a lo más llega al 12\% (no se incluye Biología, porque esta también está asociada a un perfil medio en primera opción y aparte está ubicada en la Facultad de Ciencias, la cual presenta problemas fuertes de no matrícula). Estos resultados muestran que las carreras asociadas a perfiles altos de ingreso presentan matrícula alta, contrariamente a lo observado en las carreras asociadas a perfiles de bajo ingreso.

\section{BIBLIOGRAFÍA}

\section{ARTÍ́CULOS}

Jiménez, K. y Morales, E. "Validez predictiva del Promedio de Admisión de la Universidad de Costa Rica y sus componentes". Actualidades en Psicología 23. 20092010: 21-55.

Montero, E. Indicadores de equidad para la educación superior y para la UCR en particular:desproporcionalidades que preocupan. En prensa. 2013. 
Rojas, L. "Validez predictiva de los componentes del promedio de admisión a la Universidad deCosta Rica utilizando el género y el tipo de sexo como variables control". Revista ActualidadesInvestigativas en Educación (1)13. 2013: 24.

TESIS

Cabrera, S. "Aplicación del análisis de correspondencia en estudios preliminares de mercados potenciales". [Tesis de Licenciatura]. Universidad Nacional Mayor de San Marcos, 2008.

OTROS

Oficina de Registro e Información. Proceso de Admisión [diapositivas de PowerPoint]. 15 de abril de 2013.
Programa de Pruebas Específicas. "Factores asociados a la selección e ingreso a carrera". Informe técnico. Universidad de Costa Rica, 2012.

Proyecto de Habilidades Cuantitativas. "Informe parcial del proyecto de Habilidades Cuantitativas". Informe técnico. Universidad de Costa Rica, 2012.

Reglamento del Proceso de Admisión mediante Prueba de Aptitud Académica. La Gaceta Universitaria 4776-08. 19 de febrero de 2003.

Rivera, E. "Tres de cada diez estudiantes desearía cambiar de carrera". Semanario Universidad 1982. 2013: 4-5.

Vicerrectoría de Vida Estudiantil. Sistema de Admisión UCR [diapositivas de PowerPoint]. 15 de abril de 2013. 
ANEXOS

DETALLES DEL ANÁLISIS DE CORRESPONDENCIAS

En los cuadros 3, 4 y 5 se presentan los detalles de la masa y la inercia de las carreras, obtenidos en los análisis de correspondencias. Además, se muestra la puntuación de estas en las dos dimensiones establecidas $y$ de la contribución porcentual a la inercia de las dimensiones.
En el cuadro 3 se trabajan las carreras de la Facultad de Ciencias y se observa que la carrera de Biología es la que presenta la contribución más alta en la primera dimensión, mientras que las carreras de Química y Ciencias Actuariales son las que más contribuyen en la segunda dimensión.

CUADRO 3

ESTADÍSTICOS DE LAS CARRERAS DE LA FACULTAD DE CIENCIAS OBTENIDOS EN EL ANÁLISIS DE CORRESPONDENCIAS CON EL PERFIL DE INGRESO

\begin{tabular}{|c|c|c|c|c|c|c|}
\hline \multirow{2}{*}{ CARRERA } & \multirow{2}{*}{ MASA } & \multicolumn{2}{|c|}{ PUNTUACIÓN } & \multirow{2}{*}{ INERCIA } & \multicolumn{2}{|c|}{ CONTRIBUCIÓN } \\
\hline & & DIM. 1 & DIM. 2 & & DIM. 1 & DIM. 2 \\
\hline Biología & .112 & -.923 & -.637 & .078 & .142 & . 184 \\
\hline Física & .206 & -.281 & .284 & .015 & .024 & .067 \\
\hline Meteorología & .206 & 1.454 & -.061 & .295 & .646 & .003 \\
\hline Geología & .166 & .144 & -.027 & .022 & .005 & .001 \\
\hline Matemática & .091 & .004 & .215 & .006 & .000 & .017 \\
\hline Actuariales & .083 & -.595 & -1.109 & .045 & .044 & .413 \\
\hline Química & .135 & -.832 & .760 & .083 & .139 & .315 \\
\hline TOTAL & 1.000 & & & .545 & 1.000 & 1.000 \\
\hline
\end{tabular}

Fuente: Oficina de Registro e Información de la Universidad de Costa Rica. Dim.= Dimensión

El cuadro 4 está asociado a las carreras de la Facultad de Ciencias Económicas, en el cual Economía tiene una contribución a la inercia de la primera dimensión que abarca casi la totalidad de esta, mientras que entre Estadística y Contaduría Pública presentan contribuciones muy altas a la inercia de la segunda dimensión. 
CUADRO 4

ESTADÍSTICOS DE LAS CARRERAS DE LA FACULTAD DE CIENCIAS ECONÓMICAS OBTENIDOS EN EL ANÁLISIS DE CORRESPONDENCIAS CON EL PERFIL DE INGRESO

\begin{tabular}{ccccccc}
\hline \multirow{2}{*}{ CARRERA } & \multirow{2}{*}{ MASA } & \multicolumn{2}{c}{ PUNTUACIÓN } & \multirow{2}{*}{ INERCIA } & \multicolumn{2}{c}{ CONTRIBUCIÓN } \\
\cline { 3 - 4 } & & DIM. 1 & DIM. 2 & & DIM. 1 & DIM. 2 \\
\hline Estadística & .052 & -.257 & 1.391 & .026 & .007 & .418 \\
Dir. Empresas & .459 & .111 & .182 & .008 & .012 & .063 \\
Contaduría & .209 & .526 & -.701 & .053 & .120 & .424 \\
Adm. Pública & .084 & .406 & .325 & .024 & .029 & .036 \\
Adm. & .142 & -.264 & -.072 & .016 & .020 & .003 \\
Aduanera & .053 & -2.719 & -.506 & .195 & .812 & .056 \\
Economía & & & & .322 & 1.000 & 1.000 \\
\hline TOTAL & 1.000 & & & & &
\end{tabular}

Fuente: Oficina de Registro e Información de la Universidad de Costa Rica.

Dim.= Dimensión

Los datos sobre las carreras de la Facultad de Ciencias Sociales se presentan en el cuadro 5 , en este se observa que Comunicación contribuye en un $80 \%$ a la inercia de la primera dimensión, mientras que en la segunda dimensión, las carreras que más contribuyen son Trabajo Social con un 33\% y Geografía con un $21 \%$.

CUADRO 5

ESTADÍSTICOS DE LAS CARRERAS DE LA FACULTAD DE CIENCIAS SOCIALES OBTENIDOS EN EL ANÁLISIS DE CORRESPONDENCIAS CON EL PERFIL DE INGRESO

\begin{tabular}{cccccccc}
\hline \multirow{2}{*}{ CARRERA } & MASA & \multicolumn{2}{c}{ PUNTUACIÓN } & & \multicolumn{2}{c}{ CONTRIBUCIÓN } \\
\cline { 3 - 4 } & & DIM. 1 & DIM. 2 & & DIM. 1 & DIM. 2 \\
\hline Comunicación & .094 & -2.447 & -.589 & .416 & .800 & .066 \\
Psicología & .189 & -.159 & .360 & .043 & .007 & .050 \\
Políticas & .151 & .178 & .712 & .057 & .007 & .156 \\
Trabajo Social & .097 & .026 & 1.303 & .084 & .000 & .333 \\
Historia & .103 & .632 & -.850 & .066 & .058 & .151 \\
Archivística & .058 & .583 & -.324 & .025 & .028 & .012 \\
Geografía & .091 & .705 & -1.082 & .084 & .064 & .215 \\
Antropología & .109 & .185 & -.228 & .007 & .005 & .011 \\
Sociología & .109 & .446 & -.156 & .017 & .031 & .005 \\
\hline TOTAL & 1.000 & & & .800 & 1.000 & 1.000 \\
\hline
\end{tabular}

Fuente: Oficina de Registro e Información de la Universidad de Costa Rica.

Dim.= Dimensión 
En el cuadro 6 se presentan las contribuciones de cada categoría del perfil de ingreso, en las dimensiones obtenidas en el Análisis de Correspondencias según facultad. En el análisis con la Facultad de Ciencias, la categoría bajos_opción2 y medios_opción2 tuvieron las contribuciones más altas en la primera y segunda dimensión, respectivamente. En Ciencias Sociales la mayor contribución a la primera dimensión, la realizó altos y en la segunda dimensión la realizó bajos_opción2. Finalmente, en Ciencias Económicas la categoría altos contribuyó en un $77 \%$ de la inercia de la primera dimensión, mientras que en la segunda, las mayores contribuciones fueron hechas por medios_opción2 y bajos_opción1. En el cuadro 7 se presentan los valores de la masa y la inercia de las categorías del perfil de ingreso obtenidas en los Análisis de Correspondencias según facultad.

CUADRO 6

CONTRIBUCIONES DE LAS CATEGORÍAS DEL PERFIL DE INGRESO EN LOS ANÁLISIS DE CORRESPONDENCIAS SEGÚN FACULTAD

\begin{tabular}{lcccccc}
\hline \multirow{2}{*}{ CATEGORÍA } & \multicolumn{2}{c}{ CIENCIAS } & \multicolumn{2}{c}{ CIENCIAS SOCIALES } & \multicolumn{2}{c}{ CIENCIAS ECONÓMICAS } \\
\cline { 2 - 7 } & DIM. 1 & DIM. 2 & DIM. 1 & DIM. 2 & DIM. 1 & DIM. 2 \\
\hline bajos_opcion1 & .156 & .013 & .060 & .101 & .100 & .325 \\
bajos_opcion2 & .498 & .001 & .125 & .353 & .071 & .148 \\
medios_opcion1 & .188 & .247 & .004 & .183 & .036 & .106 \\
medios_opcion2 & .069 & .691 & .017 & .287 & .023 & .336 \\
altos & .090 & .049 & .794 & .076 & .770 & .085 \\
\hline TOTAL & 1.000 & 1.000 & 1.000 & 1.000 & 1.000 & 1.000 \\
\hline
\end{tabular}

Fuente: Oficina de Registro e Información de la Universidad de Costa Rica. Dim.= Dimensión

CUADRO 7

MASA E INERCIA DE LAS CATEGORÍAS DEL PERFIL DE INGRESO EN LOS ANÁLISIS DE CORRESPONDENCIAS SEGÚN FACULTAD

\begin{tabular}{ccccccc}
\hline \multirow{2}{*}{ CATEGORÍA } & \multicolumn{2}{c}{ CIENCIAS } & \multicolumn{2}{c}{ CIENCIAS ECONÓMICAS } & \multicolumn{2}{c}{ CIENCIAS SOCIALES } \\
\cline { 2 - 7 } & MASA & INERCIA & MASA & INERCIA & MASA & INERCIA \\
\hline bajos_opcion1 & .141 & .082 & .242 & .045 & .187 & .064 \\
bajos_opcion2 & .204 & .231 & .107 & .033 & .187 & .154 \\
medios_opcion1 & .316 & .103 & .385 & .024 & .336 & .064 \\
medios_opcion2 & .237 & .074 & .216 & .034 & .190 & .102 \\
altos & .102 & .055 & .050 & .187 & .099 & .415 \\
\hline TOTAL & 1.000 & .545 & 1.000 & .322 & 1.000 & .800 \\
\hline
\end{tabular}

Fuente: Oficina de Registro e Información de la Universidad de Costa Rica. 